\title{
A Low-Cost Intelligent Mobile Indoor Environment Monitoring System
}

\author{
Peng Liu ${ }^{1, a}$, Mingjie Wang ${ }^{1, b}$, Wenkai Dong ${ }^{1, a}$, Shengqian Jiang ${ }^{1, a}$, Yanli \\ Wang $^{1, a}$, Quan Zhu ${ }^{1, a}$, Bing $\mathrm{Li}^{*, \mathrm{c}}$ and Xin Chen ${ }^{1, \mathrm{a}}$ \\ ${ }^{1}$ School of Mechanical Science and Engineering, Jilin University, Changchun 130022, China; \\ ${ }^{2}$ School of Philosophy and Society, Jilin University, Changchun 130012, China. \\ aliupeng@jlu.edu.cn, bmjwang521@163.com, “123717611@qq.com
}

Corresponding Author: Bing Li

Keywords: Environment monitoring, intelligent car, paired-sample t-test.

\begin{abstract}
Indoor air pollution is a serious problem that harms people's health. Indoor environment monitoring system could make contribution to detecting and improving indoor air quality. However, besides the high cost, the existing air quality detectors also get tricky problems with real-time monitoring and autonomous mobile which lead to a low practicability in most cases. Therefore this paper put forward an intelligent mobile indoor environment monitoring system based on Arduino's control using various sensors to detect air quality. The results showed that the data detected by our system got high positive correlation property with data from standard technical detecting apparatus and no obvious differences appeared. The system was more advanced and effective which could lead to great application value.
\end{abstract}

\section{Introduction}

Statistics data shows that people spend nearly $90 \%$ of their life time indoors. The general public always mistakenly considers that indoor air quality is better than out door's. However, study shows that the level of indoor air pollutant is twice to quintuple higher than the level of outdoor air pollutant [1]. Indoor air quality (IAQ) has been a new threat to public health.

In recent years, some small hypersensitive low-cost sensors have played a significant role in environment monitoring. The integrated wireless sensor network mainly uses wireless communication technology of ZigBee 、GSM、Bluetooth to transmit data [2-3]. Researchers connect it with the indoor air quality monitoring system's client-side on computers and smartphones. The content of air quality needed to monitor is followed: O3, PM, CO, nitrogen oxides, $\mathrm{SO} 2$, volatile organic compounds ( $\mathrm{VOC}$ ), $\mathrm{CO} 2$, methanol ( $\mathrm{HCHO}$ ), indoor temperature and humidity [4-7]. However most existing sensor network is settled, which is no autonomous mobile and real-time monitoring. Some researchers try to combine fixed-point monitoring with mobile monitoring but still failed to achieve a real autonomously mobile monitoring system [8-9]. This paper came up with a platform based on technology of smart car to make autonomously mobile monitoring system possible. Most researches on smart car were applied to reduce traffic accidents [10-11], while combining it with indoor air quality monitoring was rare.

In the rest of this paper, chapter 2 mainly introduced the system's overall structure and specific design detail; chapter 3 discussed the experiment designed to evaluate the system's availability and detailed test results would be shown in this part. The last chapter was a conclusion of this paper.

\section{System design}

Taking the fact into consideration, our system choose two chemical factors and two physical factors influencing indoor air quality to be detected as follows: CO, PM2.5, temperature and humidity. The system's overall architecture is shown in Fig.1, which is consisted of control module, smart car module, gas sensors module, and wireless communication module. 


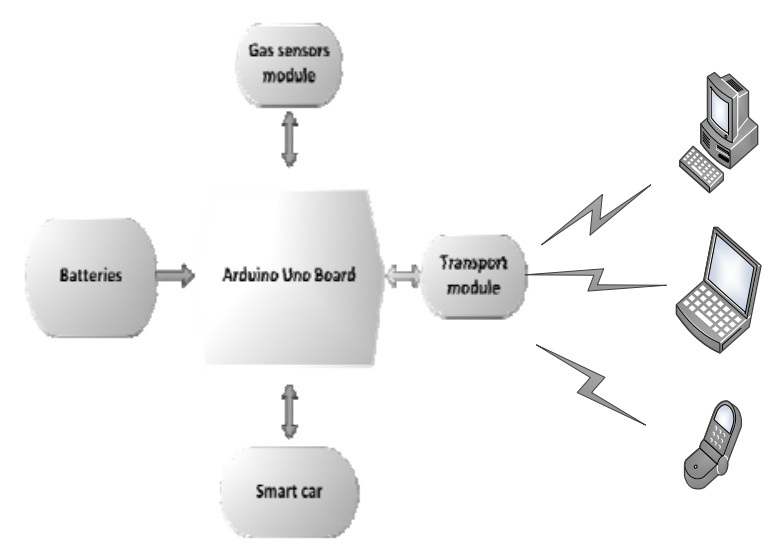

Fig. 1 The system's overall architecture

\subsection{Control module.}

The designed control module was bases on the Arduino board. Arduino is a convenient and flexible, open-source electronics prototyping platform which is easy to use, including hardware and software. The control module uses Arduino Uno as its core process data of sensors and wireless communication module and then connects to PC to give users feedback information [12].

\subsection{Smart Car module.}

Smart car module is consisted mainly by the car chassis, motor, wheels, controllers, environmental monitoring sensors, buzzer, tracking sensors, wireless communication module, motor driver board and power supply. Smart car equipped with the system modules required for the various components as well as tracking. Black trace was fixed on the white floor and infrared sensors were used to receive and transmit signal [11].

\subsection{Environment monitoring sensors module.}

Environment monitoring sensors module contains CO-sensor、PM2.5-sensor、temperature and humidity-sensor and buzzer. In our design, we use MQ-7 which is sensitive to CO to measure the level of air pollution. It has advantages of long service cycle and low cost, in addition, just a simple electric circuit could drive it [13]. Compared with other wireless communication technology, ZigBee is low-power, low-cost, which supports 65,536 network nodes. Based on IEEE802.15.4 standard low power consumption LAN protocol, ZigBee is a burgeoning short-distance wireless communication technology which is similar to Bluetooth [14]. XBee module based on ZigBee communication protocol was chosen as our wireless communication module.

\section{Methods and results of test}

Experimental design: The experiment was conducted in a resident of home in the northeast China. Given that the temperature and humidity in different points in a room at the same time change little, we detect indoor temperature and humidity for 12 hours and choose 20 sets of data for analysis. The experimenter and car should walk at the same track and the same speed. And we need to record the concentration of monitoring system and measuring instrument. 

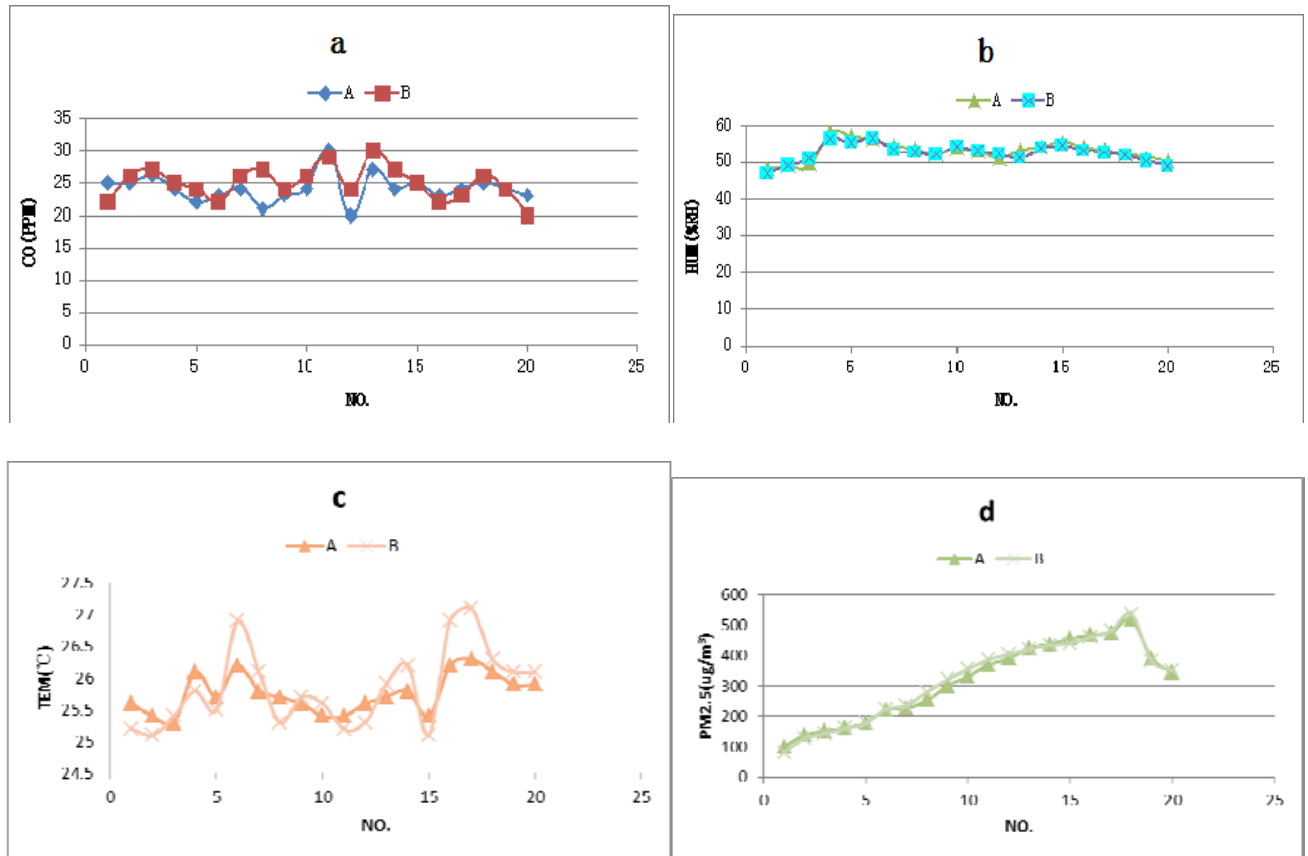

Fig.2 The results recorded for (a) CO, (b) PM2.5, (c) temperature, (d) humidity

Data collection and result: According to experimental methods above, we can record the data as A by the professional detector on the market. Environment monitoring system designed in the research records the data as B. The results recorded are shown in Fig.6.By analyzing these results, significant differences could be estimated.

Two sets of data using correlation analysis and the paired-sample t-test significant analysis were tested to evaluate reliability of the results and make decision according to value of probability $\mathrm{P}$. We adopt confidence interval of $95 \%$ and significance level of 5\% and use SPSS 19 to calculate paired correlation and value of probability $\mathrm{P}$, as well as t-test mean difference and probability $\mathrm{P}$ of paired samples, which are shown in Table 1.

Table 1The evaluation of results of t-test

\begin{tabular}{|c|c|c|c|c|c|c|}
\hline \multirow{2}{*}{ ID } & \multirow{2}{*}{$\mathrm{N}$} & \multicolumn{2}{|c|}{ The paired correlation analysis } & \multicolumn{2}{|c|}{$\begin{array}{l}\text { The paired-sample } \\
\text { significance analysis }\end{array}$} & \multirow[t]{2}{*}{ t-test } \\
\hline & & Correlation & Sig. & Mean & Sig. & \\
\hline $\mathrm{CO}$ & 20 & 0.540 & 0.014 & -8.500 & 0.150 & \\
\hline PM2.5 & 20 & 0.995 & 0.000 & -3.250 & 0.290 & \\
\hline Temperature & 20 & 0.883 & 0.000 & -0.085 & 0.324 & \\
\hline Humidity & 20 & 0.921 & 0.000 & 0.485 & 0.054 & \\
\hline
\end{tabular}

In accordance with test results, the four parameters results in two sets are accordant and paired correlation is apparent and paired-sample t-test is not apparent. The environment monitoring system has high positive correlation compared with technical detectors on market and there are no apparent differences between their detecting results [15]. The detecting results of the autonomously mobile air quality monitoring system we designed has the same tendency with those technical detectors, which means that the system gets a rational design. 


\section{Discussion and conclusion}

The design describes a smart car based on Arduino, which can move autonomously and monitor the indoor air quality real-time. And it can avoid the abuse that the operating is so complicated in the system. There are many advantages in system. It is cheaper and low-powered consumption. In addition, it doesn't need to arrange wire and can monitor real-time. It also can reflect the circumstances of air quality that people living in the room. Actual tests show that the wireless sensor networks air monitoring system which can move autonomously meet the design requirements basically. The system has the value of development and application. It should be popularized in the public. Of course, we also should study the accuracy and breadth of air quality monitoring further.

\section{References}

[1]. Kim, J.J., Jung, S.K., and Kim, J.T.: 'Wireless Monitoring of Indoor Air Quality by a Sensor Network', Indoor Built Environ, 19 (1):145-150, 2010.

[2]. Zheng, Z.B.: 'Design of distributed indoor air quality remote monitoring network', Advances in Applied Sciences and Manufacturing, Pts 1 and 2, 850-851, pp. 500-503, 2014.

[3]. Silva, J.F.M.C., Santos, D.M.S., Marques, V.C., Oliveira, K.D., Rodrigues, T.O., Texeira, R.G.F., Menezes, J.W.M., and Silva, F.D.: 'A Study of Bluetooth Application for Remote Controlling of Mobile Embedded Systems', Braz Sym Comput Syst, pp. 116-116, 2012.

[4]. Choi, G.H., Choi, G.S., and Jong, J.H.: 'A Framework for Wireless Sensor Network in Web-based Monitoring and Control of Indoor Air Quality (IAQ) in Subway Stations', 2009 2nd Ieee International Conference on Computer Science and Information Technology, Vol 2, pp. 378-382, 2009.

[5]. Kim, J.Y., Chu, C.H., and Shin, S.M.: 'ISSAQ: An Integrated Sensing Systems for Real-Time Indoor Air Quality Monitoring', Ieee Sens J, 2014, 14, (12):4230-4243

[6]. Shao, H., Sun, H., and Han, Y.Y.: 'Design and Implementation of Client for Indoor Air Quality Monitoring System Based on PC and Smartphone', 2013 6th International Conference on Intelligent Networks and Intelligent Systems (Icinis), pp. 227-230, 2013

[7]. Yu, T.C., Lin, C.C., Chen, C.C., Lee, W.L., Lee, R.G., Tseng, C.H., and Liu, S.P.: 'Wireless sensor networks for indoor air quality monitoring', Med Eng Phys, 35 (2): 231-235, 2013.

[8]. Xiang, Y., Piedrahita, R., Dick, R.P., Hannigan, M., Lv, Q., and Shang, L.: 'A Hybrid Sensor System for Indoor Air Quality Monitoring', 2013 9th Ieee International Conference on Distributed Computing in Sensor Systems (Ieee Dcoss 2013), pp. 96-104, 2013.

[9]. Jiang, Y.F., Li, K., Piedrahita, R., Xiang, Y., Tian, L., Mansata, O., Lv, Q., Dick, R.P., Hannigan, M., and Shang, L.: 'User-Centric Indoor Air-Quality Monitoring on Mobile Devices', Ai Mag, 34 (2):11-30, 2013.

[10]. Maslak, W., and Butkiewicz, B.S.: 'Autonomous vehicle with fuzzy control', 2013 Signal Processing Symposium (Sps), 2013.

[11]. Glowicki, M., and Butkiewicz, B.S.: 'Autonomous line-follower with fuzzy control', 2013 Signal Processing Symposium (Sps), 2013.

[12]. Ferdoush, S., and Li, X.R.: 'Wireless Sensor Network System Design using Raspberry Pi and Arduino for Environmental Monitoring Applications', 9th International Conference on Future Networks and Communications (Fnc'14) / the 11th International Conference on Mobile Systems and Pervasive Computing (Mobispc'14) / Affiliated Workshops, 2014, 34, pp. 103-110. 
[13]. Bhattacharjee, D., Kumar, S., Kumar, A., and Choudhury, S.: 'Design and development of wireless sensor node', International Journal on Computer Science and Engineering, 2 (07):2431-2438, 2010.

[14]. Almalkawi, I.T., Zapata, M.G., Al-Karaki, J.N., and Morillo-Pozo, J.: 'Wireless Multimedia Sensor Networks: Current Trends and Future Directions', Sensors-Basel, 10 (7): 6662-6717, 2010 .

[15]. Alshali, R.Z., Salim, N.A., Satterthwaite, J.D., and Silikas, N.: 'Post-irradiation hardness development, chemical softening, and thermal stability of bulk-fill and conventional resin-composites', J Dent, 43 (2):209-218, 2015. 